\title{
Octupole Ordering Model for the Phase IV of $\mathrm{Ce}_{x} \mathrm{La}_{1-x} \mathrm{~B}_{6}$
}

\author{
Katsunori Kubo* and Yoshio Kuramoto \\ Department of Physics, Tohoku University, Sendai 980-8578
}

(Received July 2, 2003)

An octupole ordering model is studied by the mean field theory, and its relevance to the phase IV of $\mathrm{Ce}_{x} \mathrm{La}_{1-x} \mathrm{~B}_{6}$ is discussed. The observed lattice distortion along the [111] direction is interpreted in terms of the $\Gamma_{5 g}$-type ferro-quadrupole moment induced by an antiferrooctupole ordered state with $\Gamma_{5 u}$ symmetry. The octupole model also accounts for the cusp in the magnetization as in the Néel transition, and the softening of the elastic constant $C_{44}$ below the ordering temperature. However, the internal magnetic field due to the octupole moment is smaller than the observed one by an order of magnitude. Also discussed is the possibility of a pressure induced antiferromagnetic moment in the octupole-ordered state.

KEYWORDS: rare-earth hexaboride, $\mathrm{Ce}_{x} \mathrm{La}_{1-x} \mathrm{~B}_{6}, \mathrm{NdB}_{6}, \mathrm{NpO}_{2}$, octupole ordering, elastic constant, thermal expansion, internal field, pressure induced moment

\section{Introduction}

Orbital orderings in $f$-electron systems have attracted much attention. In usual cases, an orbital-ordered phase is a quadrupole-ordered phase. However, higher multipole orderings can also be realized. Indeed, possibilities of octupole orderings in $\mathrm{NpO}_{2}{ }^{1-4)}$ and in $\mathrm{Ce}_{x} \mathrm{La}_{1-x} \mathrm{~B}_{6}{ }^{5,6)}$ have been discussed. In this paper, we study an octupole ordering model for the phase IV of $\mathrm{Ce}_{x} \mathrm{La}_{1-x} \mathrm{~B}_{6}$ paying attention to the coupling between $f$-electrons and lattice.

$\mathrm{CeB}_{6}$ has a cubic crystal structure of the $\mathrm{CaB}_{6}$-type, and $\mathrm{Ce}$ ions form a simple cubic lattice. In this material, there are three phases; the paramagnetic phase (called phase I), the antiferro-quadrupole phase (phase II) and the antiferromagnetic phase (phase III). The role of the octupole interaction in these phases has been discussed $^{6-9)}$ to explain experimental observations. In the dilute alloys $\mathrm{Ce}_{x} \mathrm{La}_{1-x} \mathrm{~B}_{6}$, the so-called phase IV was found at $x \simeq 0.75 .{ }^{10}$ ) This phase has attracted much attention. A large softening of the elastic constant $C_{44}$ just below the transition temperature $T_{\text {I-IV }}$ from the phase I to the phase IV was observed. ${ }^{11)}$ Furthermore, the magnetic susceptibility shows a cusp at $T_{\text {I-IV }}$, and the magnetization is almost isotropic at ambient pressure. ${ }^{12)}$

It was recently revealed that the lattice shrinks along the [111] direction in the phase IV. ${ }^{13,14)}$ It is probable that the softening of $C_{44}$ is due to this distortion. It may be tempting to ascribe the distortion to the ferro $\Gamma_{5 g}$-type quadrupole order. However, the quadrupole moment is not necessarily the primary order parameter. Indeed, the temperature dependence of $C_{44}$ is different from usual ferro-quadrupole-ordered materials. ${ }^{11)}$ In the phase I of $\mathrm{Ce}_{0.75} \mathrm{La}_{0.25} \mathrm{~B}_{6}$, the temperature dependence of $C_{44}$ follows the Curie-Weiss law with an antiferroquadrupole interaction, but softens drastically just below $T_{\text {I-IV }}$. The entropy changes more at the transition from the phase I to the phase IV than at the other transition from the phase IV to the phase III. ${ }^{11,15)}$ On the contrary, the entropy changes very little at the quadrupole transition in $\mathrm{CeB}_{6} \cdot{ }^{15)}$ Thus it is likely that the degeneracy of each $f$-electron state is already lifted in the phase IV.
This fact also makes it difficult for the $\Gamma_{5 g}$ quadrupoles to be the primary order parameter in the phase IV.

A large change in the internal field at $T_{\text {I-IV }}$, as probed by $\mathrm{NMR}^{16)}$ and $\mu \mathrm{SR},{ }^{17)}$ suggests strongly that the time reversal symmetry is broken in the phase IV. A pure quadrupole order is incompatible with the broken time reversal symmetry. In addition, a neutron diffraction experiment found no magnetic reflection in the phase IV. ${ }^{18}$ ) Thus, dipole moments are also unlikely to be the primary order parameter, although the time reversal symmetry is broken. Therefore, octupole moments, which break the time reversal symmetry, become a candidate for the order parameter in the phase IV. ${ }^{5,6)}$ In addition, the order parameter should have an anisotropic nature, because the cubic symmetry is broken, and the anisotropy in the magnetization develops under uniaxial pressure. ${ }^{19-21)}$ Thus the $\Gamma_{5 u}$-type octupole moment, among all octupole moments, is the most plausible candidate for the order parameter in the phase IV. We will discuss this point in detail in $\S 2$.

Kusunose and Kuramoto ${ }^{6)}$ have already pointed out using the Ginzburg-Landau (GL) theory that the $\Gamma_{5 u}$ octupole order with a finite wave number should accompany a ferro-quadrupole moment, and have suggested a possible lattice distortion. However, evaluation of the magnitude of the distortion is beyond their GL theory, because the GL theory is appropriate near the transition temperature. In this paper, we explore in much greater detail the consequence of the $\Gamma_{5 u}$ octupole order by the mean field theory, and propose that the lattice distortion and the softening of the elastic constant $C_{44}$ are due to the order of the $\Gamma_{5 u}$ octupole moment. Some of the results in this paper were reported in a recent paper. ${ }^{22)} \mathrm{In}$ this paper, we describe the details of the calculation, and add some new results, especially, the quadrupole susceptibilities.

This paper is organized as follows. In $\S 2$, we introduce an octupole ordering model and solve this model by using the mean field theory. In $\S 3, \S 4$ and $\S 5$, we discuss the magnetization, elastic constants and lattice distortion, respectively, in the octupole-ordered state. In $\S 6$, we

*E-mail: katukubo@cmpt.phys.tohoku.ac.jp 
estimate the internal field from the octupole moment. In $\S 7$, we discuss a possible dipole moment under uniaxial pressure in the octupole-ordered state. The last section is devoted to a summary.

\section{Mean Field Theory}

The crystalline electric field $(\mathrm{CEF})$ ground state of $\mathrm{Ce}^{3+}(J=5 / 2)$ in $\mathrm{Ce}_{x} \mathrm{La}_{1-x} \mathrm{~B}_{6}$ is the $\Gamma_{8}$ quartet. ${ }^{23-25)}$ The excited level $\Gamma_{7}$ lies about $500 \mathrm{~K}$ from the $\Gamma_{8}$ level and is neglected. The $\Gamma_{8}$ states are represented in terms of eigenstates of $J_{z}$ as

$$
\begin{aligned}
& |+\uparrow\rangle=\sqrt{5 / 6}|+5 / 2\rangle+\sqrt{1 / 6}|-3 / 2\rangle, \\
& |+\downarrow\rangle=\sqrt{5 / 6}|-5 / 2\rangle+\sqrt{1 / 6}|+3 / 2\rangle, \\
& |-\uparrow\rangle=|+1 / 2\rangle, \\
& |-\downarrow\rangle=|-1 / 2\rangle .
\end{aligned}
$$

Within the $\Gamma_{8}$ quartet, the number of independent multipolar moments is 15 , and active octupole moments have either $\Gamma_{2 u}, \Gamma_{4 u}$ or $\Gamma_{5 u}$ symmetry. ${ }^{7)}$

We first discuss the properties of the $\Gamma_{2 u}$ and $\Gamma_{4 u}$ moments. The $\Gamma_{4 u}$-type octupole moments accompany dipole moments, because dipole moments have the same symmetry $\Gamma_{4 u} .{ }^{7)}$ Thus the $\Gamma_{4 u}$ octupole moments are unlikely to be the order parameter in the phase IV. The $\Gamma_{2 u}$-type octupole moment, as proposed in ref. 5 , is pseudoscalar, and eigenstates of the $\Gamma_{2 u}$ moment have the cubic symmetry. Hence it does not accompany quadrupole moments, and it seems difficult to explain the lattice distortion in the phase IV by the $\Gamma_{2 u}$ octupole orderings. In the followings, we study the properties of $\Gamma_{5 u}$ octupole moments, and discuss their possibility as the order parameter of the phase IV.

Let us introduce pseudospins $\boldsymbol{\sigma}$ and $\boldsymbol{\tau}$ to describe the $\Gamma_{8}$ quartet:

$$
\begin{gathered}
\tau^{z}\left| \pm \sigma^{z}\right\rangle= \pm\left| \pm \sigma^{z}\right\rangle, \\
\sigma^{z}\left|\tau^{z} \uparrow\right\rangle=+\left|\tau^{z} \uparrow\right\rangle, \sigma^{z}\left|\tau^{z} \downarrow\right\rangle=-\left|\tau^{z} \downarrow\right\rangle,
\end{gathered}
$$

and the transverse components which flip the pseudospins. The relevant multipole moments are given with the notation $(\alpha, \beta, \gamma)=(x, y, z),(y, z, x)$ or $(z, x, y)$ by

$$
\begin{aligned}
\boldsymbol{J} & =\frac{7}{6}\left[\boldsymbol{\sigma}+\frac{4}{7}\left(\eta^{+} \sigma_{x}, \eta^{-} \sigma_{y}, \tau_{z} \sigma_{z}\right)\right], \\
O_{2}^{0} & =\frac{1}{\sqrt{3}}\left(2 J_{z}^{2}-J_{x}^{2}-J_{y}^{2}\right)=\frac{8}{\sqrt{3}} \tau^{z}, \\
O_{2}^{2} & =J_{x}^{2}-J_{y}^{2}=\frac{8}{\sqrt{3}} \tau^{x}, \\
O_{\beta \gamma} & =2 \overline{J_{\beta} J_{\gamma}}=\frac{2}{\sqrt{3}} \tau^{y} \sigma^{\alpha}, \\
T_{\alpha}^{5 u} & =\frac{1}{2 \sqrt{3}}\left(\overline{J_{\alpha} J_{\beta}^{2}}-\overline{J_{\gamma}^{2} J_{\alpha}}\right),
\end{aligned}
$$

and

$$
\left(T_{x}^{5 u}, T_{y}^{5 u}, T_{z}^{5 u}\right)=\left(\zeta^{+} \sigma^{x}, \zeta^{-} \sigma^{y}, \tau^{x} \sigma^{z}\right)
$$

where bars on the products represent normalized symmetrization, e.g., $\overline{J_{x} J_{y}^{2}}=\left(J_{x} J_{y}^{2}+J_{y} J_{x} J_{y}+J_{y}^{2} J_{x}\right) / 3$, and

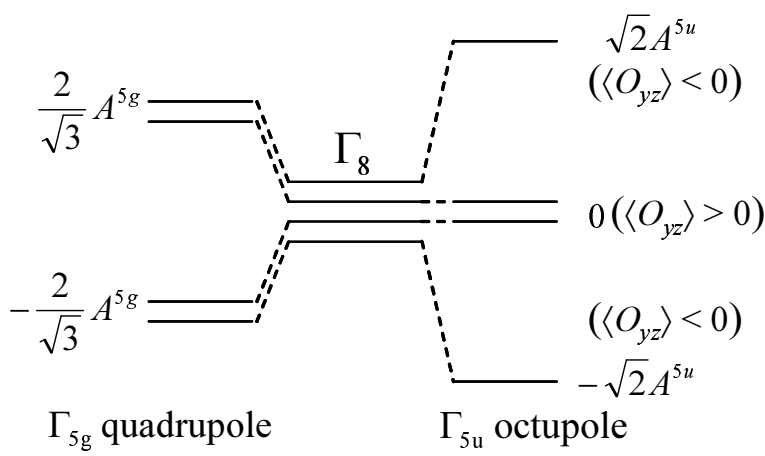

Fig. 1. The level scheme in the $\Gamma_{5 u}$ interaction (right) and in the $\Gamma_{5 g}$ interaction (left). The quantities $A^{5 u}$ and $A^{5 g}$ are the octupolar and quadrupolar mean fields.

we have introduced the notations

$$
\begin{aligned}
\eta^{ \pm} & =\frac{1}{2}\left( \pm \sqrt{3} \tau^{x}-\tau^{z}\right), \\
\zeta^{ \pm} & =-\frac{1}{2}\left(\tau^{x} \pm \sqrt{3} \tau^{z}\right) .
\end{aligned}
$$

\subsection{Level Scheme}

Before introducing a octupole ordering model on a lattice, we discuss the 'easy axis' of the $\Gamma_{5 u}$ octupole moment under a fictitious octupole field $\boldsymbol{A}^{5 u}=\left(A_{x}^{5 u}, A_{y}^{5 u}, A_{z}^{5 u}\right)$. It is sufficient to consider highsymmetry directions of $\boldsymbol{A}^{5 u}$. With $\left|\boldsymbol{A}^{5 u}\right|=1$, the four eigenvalues $(\mathrm{Ev})$ of relevant cases are given as follows:

(i) $\boldsymbol{A}^{5 u} \|(0,0,1): \operatorname{Ev}\left[T_{z}^{5 u}\right]= \pm 1$ (both doubly degenerate);

(ii) $\boldsymbol{A}^{5 u} \|(1,1,0): \operatorname{Ev}\left[\left(T_{x}^{5 u}+T_{y}^{5 u}\right) / \sqrt{2}\right]= \pm(\sqrt{3} \pm 1) / 2$;

(iii) $\boldsymbol{A}^{5 u} \|(1,1,1): \operatorname{Ev}\left[\left(T_{x}^{5 u}+T_{y}^{5 u}+T_{z}^{5 u}\right) / \sqrt{3}\right]= \pm \sqrt{2}$ and 0 (doubly degenerate).

Case (iii) gives the largest eigenvalue, and thus the easy axis is along the [111] direction and equivalent ones.

In the mean field theory, the fictitious octupole field represents the intersite interaction. When the intersite interaction is isotropic, a state with $\left(\left|\left\langle T_{x}^{5 u}\right\rangle\right|,\left|\left\langle T_{y}^{5 u}\right\rangle\right|,\left|\left\langle T_{z}^{5 u}\right\rangle\right|\right) \|(1,1,1)$ is the most stable. We note that the operator $T_{x}^{5 u}+T_{y}^{5 u}+T_{z}^{5 u}$ commutes with $O_{y z}+O_{z x}+O_{x y}$, and these two operators can be diagonalized simultaneously. On the right side of Fig. 1, we illustrate the level scheme in octupole field $\boldsymbol{A}^{5 u}=A^{5 u}(1,1,1) / \sqrt{3}$. The $\Gamma_{8}$ level splits into three levels. Not only the time reversal symmetry, but also the cubic symmetry is broken. The multipolar moments in the ground and highest states are

$$
\begin{aligned}
& \left\langle T_{x}^{5 u}\right\rangle=\left\langle T_{y}^{5 u}\right\rangle=\left\langle T_{z}^{5 u}\right\rangle=\mp \sqrt{2 / 3}, \\
& \left\langle O_{y z}\right\rangle=\left\langle O_{z x}\right\rangle=\left\langle O_{x y}\right\rangle=-2 / 3,
\end{aligned}
$$

and the others are zero. Thus, ferro, antiferro and other collinearly ordered states with $\left(\left\langle T_{x}^{5 u}\right\rangle,\left\langle T_{y}^{5 u}\right\rangle,\left\langle T_{z}^{5 u}\right\rangle\right) \|$ $(1,1,1)$ have a homogeneous $\Gamma_{5 g}$ moment. With the quadrupole-strain coupling, the crystal should distort along [111].

It is obvious that a $\Gamma_{5 g}$-type ferro-quadrupole interaction alone can lead to $\left\langle O_{y z}\right\rangle=\left\langle O_{z x}\right\rangle=\left\langle O_{x y}\right\rangle \neq 0$. The Kramers degeneracy remains in this case. The en- 


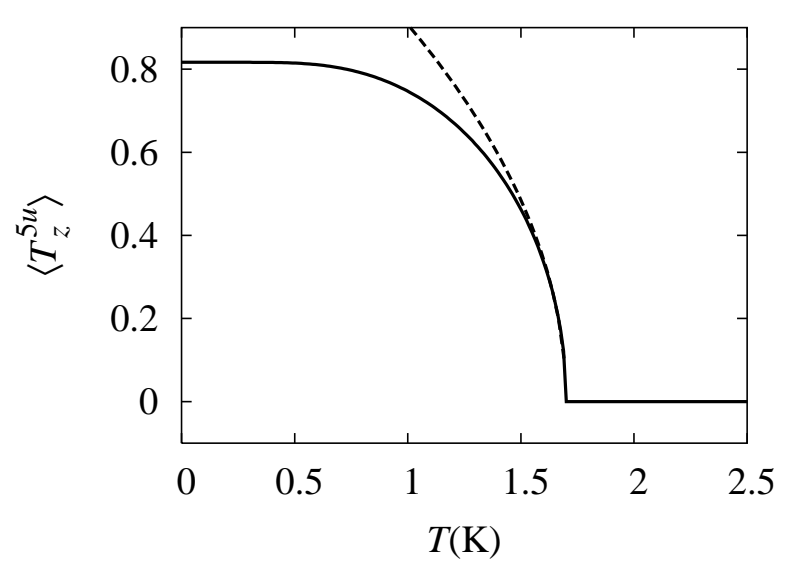

Fig. 2. Temperature dependence of the antiferro-octupole moment $\left\langle T_{z}^{5 u}\right\rangle\left(=\left\langle T_{x}^{5 u}\right\rangle=\left\langle T_{y}^{5 u}\right\rangle\right)$. The dashed line is the asymptotic behavior $\sqrt{2}\left(1-T / T_{5 u}\right)^{1 / 2}$ at $T \simeq T_{5 u}$.

ergy level splits into two levels, and the ground state is twofold degenerate as shown on the left side of Fig. 1.

\subsection{Antiferro-Octupole Ordering Model}

We proceed to consider the $\Gamma_{8}$ quartets on a simple cubic lattice. From the above discussion, we find that the order parameter which breaks the time reversal symmetry and induces quadrupole moments, but which accompanies no dipole moment is only the $\Gamma_{5 u}$ octupole moment. However, we cannot determine the periodicity of the $\Gamma_{5 u}$-ordered state in the phase IV only from the above consideration, because any collinearly ordered state with $\left(\left\langle T_{x}^{5 u}\right\rangle,\left\langle T_{y}^{5 u}\right\rangle,\left\langle T_{z}^{5 u}\right\rangle\right) \|(1,1,1)$ has a uniform $\Gamma_{5 g}$ moment. In the present study, we consider a G-type (staggered) antiferro-octupole order as the simplest example of $\Gamma_{5 u}$ orders. The importance of the $\Gamma_{5 u}$ nearest-neighbor interaction in causing the change from the phase III to the phase III', even with a weak magnetic field, was pointed out in ref. 6 . We consider only this nearest-neighbor interaction, and take the following model:

$$
\mathcal{H}=I^{5 u} \sum_{(i, j)} \sum_{\alpha=x, y, z} T_{\alpha i}^{5 u} T_{\alpha j}^{5 u},
$$

where $(i, j)$ denotes a nearest-neighbor pair. We study this Hamiltonian by the mean field theory, and choose the value of $I^{5 u}$ so as to reproduce the transition temperature $T_{\text {I-IV }}$ in $\mathrm{Ce}_{0.75} \mathrm{La}_{0.25} \mathrm{~B}_{6}$, i.e., $T_{5 u}=6 I^{5 u}=1.7 \mathrm{~K}$. In carrying out the mean field theory, we assume the G-type two sublattice structure, but do not assume a particular solution, e.g., $\left\langle T_{x}^{5 u}\right\rangle=\left\langle T_{y}^{5 u}\right\rangle=\left\langle T_{z}^{5 u}\right\rangle$. When we obtain several self-consistent solutions, we choose the one which has the lowest free energy.

The temperature dependences of $\left\langle T_{z}^{5 u}\right\rangle$ and $\left\langle O_{x y}\right\rangle$ are shown in Figs. 2 and 3. The solution obtained has the antiferro-octupole order with $\left\langle T_{x}^{5 u}\right\rangle=\left\langle T_{y}^{5 u}\right\rangle=\left\langle T_{z}^{5 u}\right\rangle$, accompanying the ferro-quadrupole moment $\left\langle O_{y z}\right\rangle=$ $\left\langle O_{z x}\right\rangle=\left\langle O_{x y}\right\rangle$. At $T \simeq T_{5 u}$, we obtain $\left\langle T_{z}^{5 u}\right\rangle \propto(1-$ $\left.T / T_{5 u}\right)^{1 / 2}$ as a consequence of the mean field theory. On the other hand, the quadrupole moment behaves as $\left\langle O_{x y}\right\rangle \propto\left(1-T / T_{5 u}\right)$, which indicates the feature of the induced order parameter. ${ }^{6)}$ We also find other equivalent

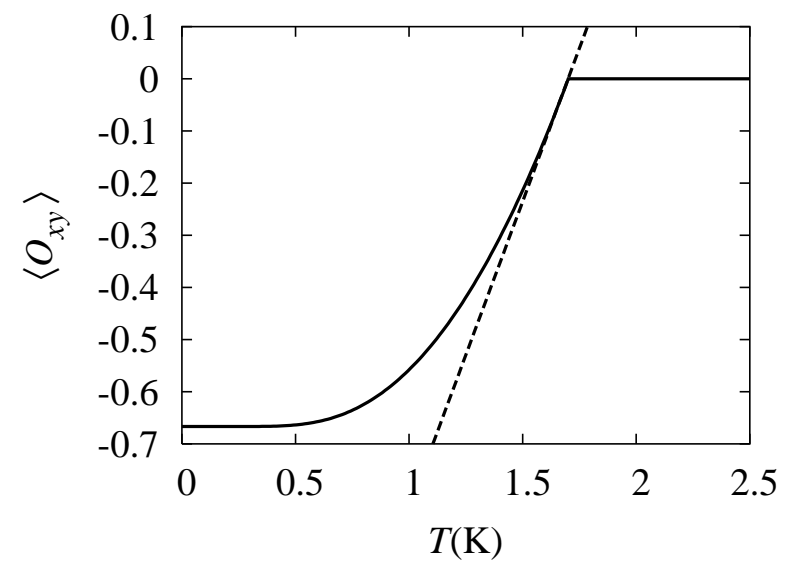

Fig. 3. Temperature dependence of the ferro-quadrupole moment $\left\langle O_{x y}\right\rangle\left(=\left\langle O_{y z}\right\rangle=\left\langle O_{z x}\right\rangle\right)$. The dashed line is the asymptotic behavior $-2\left(1-T / T_{5 u}\right)$ at $T \simeq T_{5 u}$.

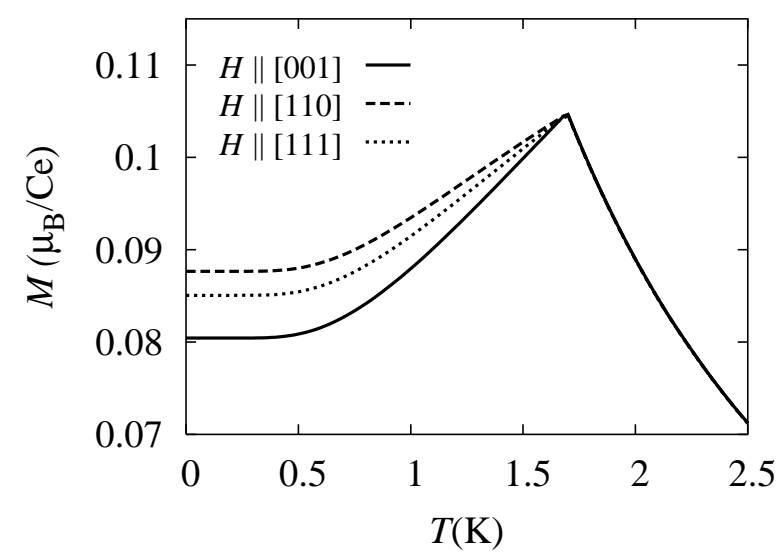

Fig. 4. Temperature dependence of the magnetization in magnetic field $H=0.2 \mathrm{~T}$ along various directions. The domains are selected by the magnetic field directions.

solutions: $\left(\left\langle T_{\alpha}^{5 u}\right\rangle,\left\langle T_{\beta}^{5 u}\right\rangle,\left\langle T_{\gamma}^{5 u}\right\rangle,\left\langle O_{\beta \gamma}\right\rangle,\left\langle O_{\gamma \alpha}\right\rangle,\left\langle O_{\alpha \beta}\right\rangle\right)=$ $( \pm B, \pm B, \mp B,+C,+C,-C)$ with $(\alpha, \beta, \gamma)=(x, y, z)$, $(y, z, x)$ or $(z, x, y)$, where quantities $B$ and $C$ are positive and depend on temperature. The degeneracy of these solutions can be lifted by a magnetic field or pressure. In the following sections, we choose a domain in which the free energy is minimized.

\section{Magnetization}

A magnetic field can lift the degeneracy of the domains. For $\boldsymbol{H} \|$ [111], the (111) domain given by eqs. (2.15) and (2.16) is not the most stable one. Instead, a state where two of $\left\langle O_{\alpha \beta}\right\rangle$ 's have positive values, and the other has a negative value is stabilized. For $\boldsymbol{H} \|[110],\left\langle J_{x}\right\rangle$ and $\left\langle J_{y}\right\rangle$ have positive values, and a state with $\left\langle O_{x y}\right\rangle>0$ is realized. On the other hand, all the domains obtained in $\S 2$ have the same energy under $\boldsymbol{H} \|[001]$.

In Fig. 4, we show the temperature dependence of the magnetization in magnetic field $H=0.2 \mathrm{~T}$ along the three high-symmetry directions. The magnetization has a cusp at the transition temperature $T_{5 u}$. The presence of a cusp is consistent with experimental observa- 


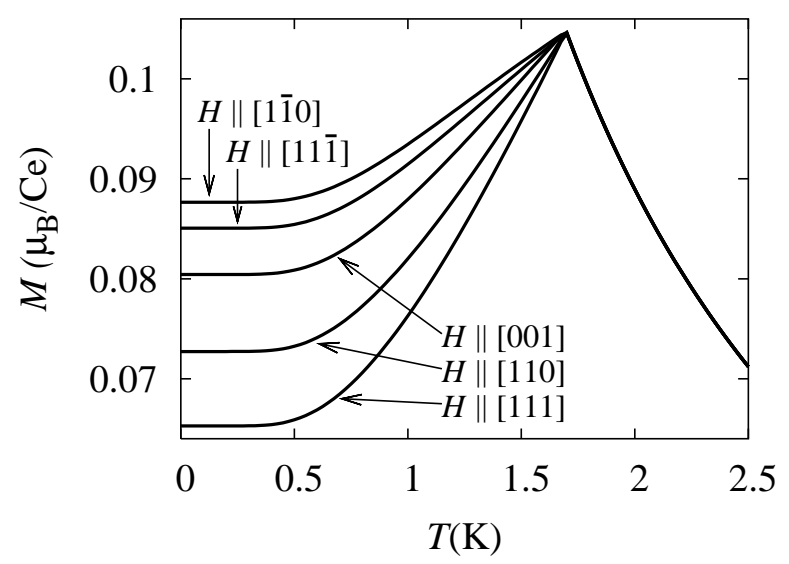

Fig. 5. Temperature dependence of the magnetization in the single domain with $\left\langle T_{x}^{5 u}\right\rangle=\left\langle T_{y}^{5 u}\right\rangle=\left\langle T_{z}^{5 u}\right\rangle$ in magnetic field $H=0.2 \mathrm{~T}$ along various directions.

tions. ${ }^{12,19-21)}$ Very recently, magnetization under uniaxial pressure $p=100 \mathrm{MPa}$ along the [111] direction has been measured at $H=0.2 \mathrm{~T}$ and $H=0.4 \mathrm{~T} .{ }^{21)}$ The observed magnetization is anisotropic and $M_{[1 \overline{1} 0]}>$ $M_{[001]}>M_{[111]}$. It seems that the system under the uniaxial pressure has a single domain. In order to deal with this situation, we have computed the temperature dependence of the magnetization assuming a single domain with $\left\langle T_{x}^{5 u}\right\rangle=\left\langle T_{y}^{5 u}\right\rangle=\left\langle T_{z}^{5 u}\right\rangle\left(\left\langle O_{y z}\right\rangle=\left\langle O_{z x}\right\rangle=\left\langle O_{x y}\right\rangle\right)$ in magnetic field $H=0.2 \mathrm{~T}$. The results are shown in Fig. 5. The anisotropy derived by our model is consistent with the experimental observation.

On the other hand, a previous experiment without an external stress, the magnetization below $T_{\text {I-IV }}$ is almost isotropic. ${ }^{12)}$ The observed isotropy may be due to a multi-domain structure. Our model have four equivalent solutions in the absence of a magnetic field. If these four domains are distributed with the same probability, the magnetic susceptibility is isotropic, because the cubic symmetry is effectively recovered in the multidomain state. The anisotropy in the magnetization under $p \|[001]$ was also reported. ${ }^{19,20)}$ It is not easy to explain this anisotropy, because the four domains in our model are equivalent under $p \|[001]$ and it seems that a single domain state is not realized under $p \|$ [001].

We have also calculated the magnetization in the case of ferro-octupole interaction. The result is qualitatively similar to that in the antiferro-octupole case; the magnetization has a cusp, and the anisotropy in the single domain with $\left\langle T_{x}^{5 u}\right\rangle=\left\langle T_{y}^{5 u}\right\rangle=\left\langle T_{z}^{5 u}\right\rangle$ is the same as that of the antiferro-octupole case. Therefore, it is difficult to exclude the possibility of the ferro-octupole ordering in the phase IV from the magnetization alone.

For comparison, we show the magnetization of a model in which only the $\Gamma_{5 g}$ ferro-quadrupole interaction is present in Fig. 6. The interaction between quadrupole moments is chosen so as to reproduce the transition temperature $T_{\mathrm{I}-\mathrm{IV}}=1.7 \mathrm{~K}$. In this calculation, the domains are determined by the direction of a magnetic field. We cannot fix a domain in this case, because the ground state is continuously degenerate in the absence of a magnetic field. For example, the state with $\left\langle O_{y z}\right\rangle=\left\langle O_{z x}\right\rangle=\left\langle O_{x y}\right\rangle$

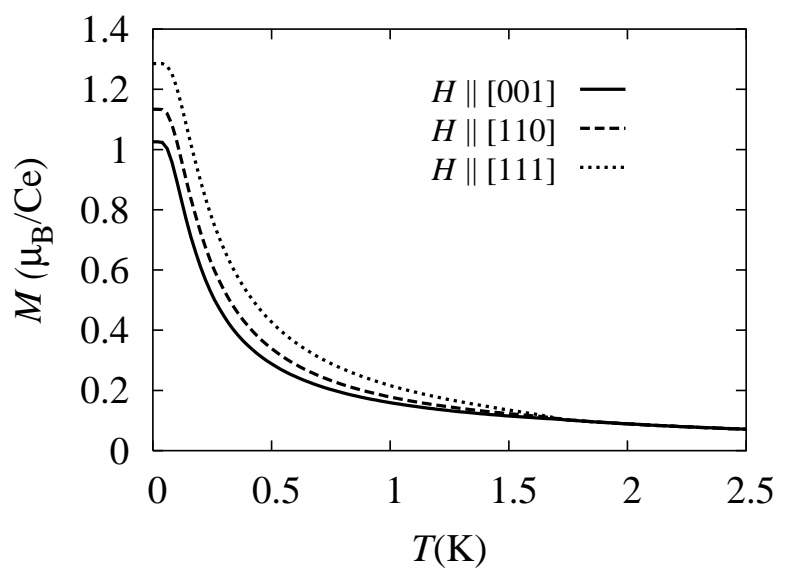

Fig. 6. Temperature dependence of the magnetization in magnetic field $H=0.2 \mathrm{~T}$ along various directions for the case where the $\Gamma_{5 g}$ quadrupole moment is the primary order parameter.

is not even metastable in $\boldsymbol{H} \|$ [001]. The magnetization only slightly changes at the quadrupole transition temperature, and does not have a cusp. Thus it is unlikely that the primary order parameter in the phase IV is the ferro-quadrupole moment.

\section{Elastic Constant}

In ferro-quadrupole-ordered materials such as TmZn, ${ }^{26)}$ the elastic constant often shows a large softening, following the Curie-Weiss law, as the transition temperature is approached from above. However, the softening of $C_{44}$ above $T_{\text {I-IV }}$ is very small in $\mathrm{Ce}_{0.75} \mathrm{La}_{0.25} \mathrm{~B}_{6}$, and a large softening is observed just below $T_{\text {I-IV. }}{ }^{11)}$ Moreover, an antiferro-quadrupole interaction $g_{\Gamma_{5}}^{\prime}=-2 \mathrm{~K}$ is deduced by a fitting of the temperature dependence of $C_{44}$ above $T_{\text {I-IV }}$. Thus it is difficult to ascribe this softening to a ferro-quadrupole interaction. In this section, we consider the elastic constant in the octupole ordering model. We do not include the quadrupole-quadrupole interaction $g_{\Gamma_{5}}^{\prime}$ for simplicity.

The elastic energy associated with the quadrupole moments is given per unit volume by

$$
E=\sum_{\Gamma \gamma}\left(\frac{1}{2} \epsilon_{\Gamma \gamma}^{2} C_{\Gamma}^{(0)}+g_{\Gamma} \sum_{i} \epsilon_{\Gamma \gamma} O_{\Gamma \gamma i}\right),
$$

where $\epsilon_{\Gamma \gamma}$ is the strain tensor, $g_{\Gamma}$ is the magneto-elastic coupling constant, and $C_{\Gamma}^{(0)}$ is the bare elastic constant. The second sum runs over Ce sites $i$ in a unit volume. The second derivative of the free energy with respect to $\epsilon_{\Gamma \gamma}$ gives the elastic constant $C_{\Gamma},{ }^{27)}$ which is given by

$$
C_{\Gamma}=C_{\Gamma}^{(0)}-N g_{\Gamma}^{2} \chi_{\Gamma},
$$

where $N$ is the number of Ce ions in the unit volume and 


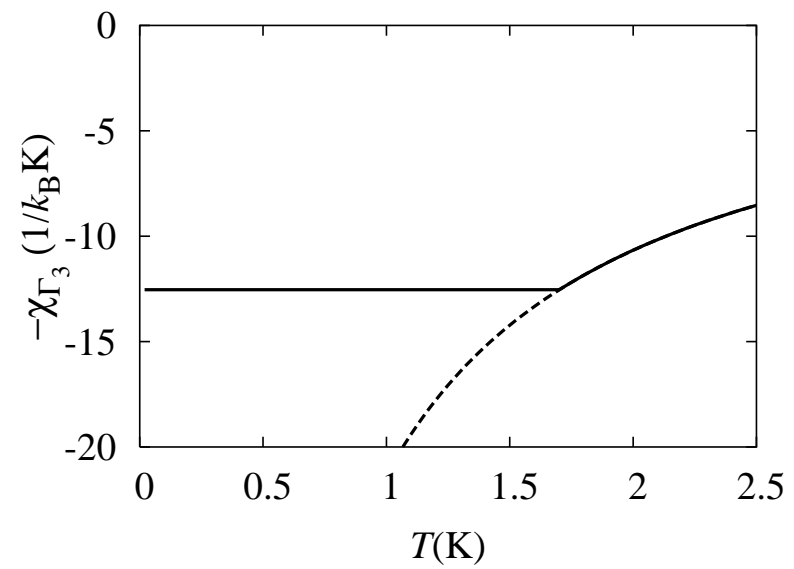

Fig. 7. Temperature dependence of the quadrupole susceptibility $\chi_{\Gamma_{3}}$ (solid line). The Curie law behavior $\chi_{\Gamma_{3}}^{\mathrm{C}}=64 /\left(3 k_{\mathrm{B}} T\right)$ at high temperatures is also shown (dashed line).

$\chi_{\Gamma}$ is the quadrupole susceptibility defined by

$$
\begin{aligned}
\chi_{\Gamma}= & -\left.\frac{\mathrm{d}\left\langle O_{\Gamma \gamma}\right\rangle}{\mathrm{d}\left(g_{\Gamma} \epsilon_{\Gamma \gamma}\right)}\right|_{g_{\Gamma} \epsilon_{\Gamma \gamma}=0} \\
= & \left.\frac{1}{k_{\mathrm{B}} T} \sum_{n} p_{n}\left[\left\langle n\left|O_{\Gamma \gamma}\right| n\right\rangle-\left\langle O_{\Gamma \gamma}\right\rangle_{0}\right] \frac{\mathrm{d} E_{n}}{\mathrm{~d}\left(g_{\Gamma} \epsilon_{\Gamma \gamma}\right)}\right|_{g_{\Gamma} \epsilon_{\Gamma \gamma}=0} \\
& -\left.\sum_{n} p_{n} \frac{\mathrm{d}\left\langle n\left|O_{\Gamma \gamma}\right| n\right\rangle}{\mathrm{d}\left(g_{\Gamma} \epsilon_{\Gamma \gamma}\right)}\right|_{g_{\Gamma} \epsilon_{\Gamma \gamma}=0},
\end{aligned}
$$

where $E_{n}$ and $|n\rangle$ are the eigenvalue and eigenstate of the total Hamiltonian, and $\langle\cdots\rangle_{0}$ denotes the expectation value without the strain. The occupation probability $p_{n}$ is given by

$$
p_{n}=\frac{e^{-E_{n} / k_{\mathrm{B}} T}}{\sum_{m} e^{-E_{m} / k_{\mathrm{B}} T}} .
$$

The elastic constants, strain tensors and quadrupole moments with $\Gamma_{3}$ and $\Gamma_{5}$ symmetries are given as follows:

$$
\begin{aligned}
C_{\Gamma_{3}} & =\left(C_{11}-C_{12}\right) / 2, \\
C_{\Gamma_{5}} & =4 C_{44}, \\
\epsilon_{\Gamma_{3} u} & =\epsilon_{u}=\left(2 \epsilon_{z z}-\epsilon_{x x}-\epsilon_{y y}\right) / \sqrt{3}, \\
\epsilon_{\Gamma_{3} v} & =\epsilon_{v}=\epsilon_{x x}-\epsilon_{y y}, \\
\epsilon_{\Gamma_{5} \alpha \beta} & =\epsilon_{\alpha \beta}, \\
O_{\Gamma_{3} u} & =O_{2}^{0}, \\
O_{\Gamma_{3} v} & =O_{2}^{2}, \\
O_{\Gamma_{5} \alpha \beta} & =O_{\alpha \beta} .
\end{aligned}
$$

We calculate $\chi_{\Gamma}$ by differentiating $\left\langle O_{\Gamma \gamma}\right\rangle$ with respect to $g_{\Gamma} \epsilon_{\Gamma \gamma}$ numerically. In Figs. 7 and 8, we show the temperature dependence of $\chi_{\Gamma_{3}}$ and $\chi_{\Gamma_{5}}$. Above the transition temperature $T_{5 u}$, the octupole interaction $I^{5 u}$ does not influence $\chi_{\Gamma_{3}}$ and $\chi_{\Gamma_{5}}$, because the strain $\epsilon_{\Gamma \gamma}$ does not induce octupole moments in the non-ordered state. The quadrupole susceptibility $\chi_{\Gamma_{5}}$ jumps at $T_{5 u}$ due to the

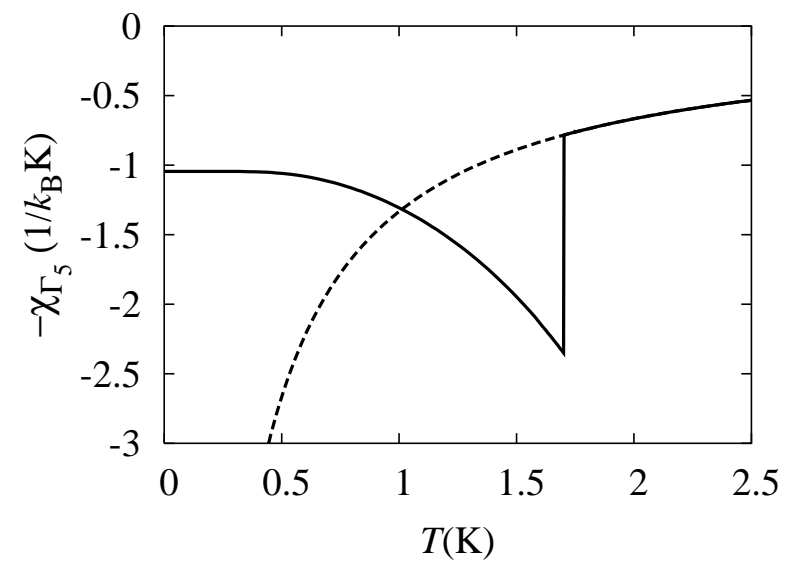

Fig. 8. Temperature dependence of the quadrupole susceptibility $\chi_{\Gamma_{5}}$ (solid line). The Curie law behavior $\chi_{\Gamma_{5}}^{\mathrm{C}}=4 /\left(3 k_{\mathrm{B}} T\right)$ at high temperatures is also shown (dashed line).

octupole order. Although the mean field theory gives a finite jump at $T_{5 u}$, fluctuations beyond the mean field theory should lead to divergence of $\chi_{\Gamma_{5}}$ at $T_{5 u}$.

For further understanding of the jump obtained above, we explore $\chi_{\Gamma_{5}}$ at $T \simeq T_{5 u}$ analytically. The mean field Hamiltonian for the A-sublattice with finite $\epsilon_{x y}$ without magnetic field is given by

$$
\mathcal{H}_{\mathrm{MF}}=-6 I^{5 u} \sum_{\alpha}\left\langle T_{\alpha}^{5 u}\right\rangle T_{\alpha}^{5 u}+g_{\Gamma_{5}} \epsilon_{x y} O_{x y} .
$$

For $T<T_{5 u}$ and $\epsilon_{x y} \neq 0$, we have $\left\langle T_{x}^{5 u}\right\rangle=\left\langle T_{y}^{5 u}\right\rangle \neq$ $\left\langle T_{z}^{5 u}\right\rangle$. We determine these quantities self-consistently. We obtain $E_{n}$ and $|n\rangle$ of $\mathcal{H}_{\mathrm{MF}}$ by perturbation theory and calculate $\chi_{\Gamma_{5}}$ by using eq. (4.20). At $T \simeq T_{5 u}$, we obtain

$$
\chi_{\Gamma_{5}}=\frac{4}{3 k_{\mathrm{B}} T}-\frac{2 \sqrt{6} I^{5 u}}{k_{\mathrm{B}} T} \chi_{x}^{5 u-x y} h
$$

where

$$
h=-\frac{E_{g}}{k_{\mathrm{B}} T}=\frac{2 \sqrt{6} I^{5 u}}{k_{\mathrm{B}} T}\left\langle T_{x}^{5 u}+T_{y}^{5 u}+T_{z}^{5 u}\right\rangle_{0}
$$

with $E_{g}$ the mean field ground state energy per Ce ion. We have also introduced the octupole susceptibility with respect to the strain $\epsilon_{x y}$, which is defined by

$$
\chi_{\alpha}^{5 u-x y}=-\left.\frac{\mathrm{d}\left\langle T_{\alpha}^{5 u}\right\rangle}{\mathrm{d}\left(g_{\Gamma_{5}} \epsilon_{x y}\right)}\right|_{g_{\Gamma_{5}} \epsilon_{x y}=0} .
$$

By symmetry, we obtain

$$
\chi_{x}^{5 u-x y}=\chi_{y}^{5 u-x y} .
$$

At $T>T_{5 u}$, we have $h=0$ and $\chi_{\alpha}^{5 u-x y}=0$, and then only the first term in eq. (4.31), i.e, the Curie term remains finite. The second term in eq. (4.31) becomes finite at $T<T_{5 u}$. The octupole susceptibility satisfies the equation

$$
h^{2}\left(\begin{array}{ll}
2 & 1 \\
1 & 5
\end{array}\right)\left(\begin{array}{c}
\chi_{z}^{5 u-x y} \\
\chi_{x}^{5 u-x y}
\end{array}\right)=-\frac{6 \sqrt{6}}{k_{\mathrm{B}} T} h\left(\begin{array}{l}
0 \\
1
\end{array}\right) .
$$


When $h \neq 0$, i.e, $T<T_{5 u}$, we obtain

$$
\begin{aligned}
\chi_{z}^{5 u-x y} & =-\frac{1}{2} \chi_{x}^{5 u-x y}, \\
\chi_{x}^{5 u-x y} & =-\frac{1}{k_{\mathrm{B}} T} \frac{4 \sqrt{6}}{3} h^{-1} .
\end{aligned}
$$

Therefore $\chi_{x}^{5 u-x y}$ diverges at $T_{\mathrm{I}-\mathrm{IV}}$, and the second term in eq. (4.31) becomes finite just below $T_{5 u}$. The change of the susceptibility $\Delta \chi_{\Gamma_{5}}$ at $T_{5 u}$ is given by

$$
\Delta \chi_{\Gamma_{5}}=\frac{8}{3 k_{\mathrm{B}} T_{5 u}} .
$$

Experimentally, $C_{44}$ remains finite at $T_{\mathrm{I}-\mathrm{IV}}$. Moreover, $C_{44}$ becomes smaller as temperature is lowered below $T_{\text {I-IV. }}$ In general, the second-order transition involving the lattice distortion along [111] leads to divergence of $C_{44}$ at the transition temperature. Thus the observed behavior of the elastic constant seems to be influenced by the experimental setup, and may not be intrinsic to $C_{44}$. One possible reason for this behavior is that the large ultrasonic absorption accompanying softening makes it difficult to determine the elastic constant accurately. Another possibility is the mode mixing of the sound wave. To determine $C_{44}$, sound wave with wave vector $\boldsymbol{k} \|$ [001] and polarization $\boldsymbol{u} \|$ [100] was used in the experiment. ${ }^{11)}$ However, this mode is not a normal mode below $T_{\mathrm{I}-\mathrm{IV}}$, because the cubic symmetry is broken. It is desirable to measure the longitudinal ultrasound velocity with $\boldsymbol{k} \|$ [111] which is expected to reflect the anomaly in $C_{44}$ directly, because this mode remains a normal mode even in the trigonal symmetry $\left(\left\langle O_{y z}\right\rangle=\left\langle O_{z x}\right\rangle=\left\langle O_{x y}\right\rangle \neq 0\right)$.

\section{Lattice Distortion}

We now discuss the lattice distortion due to the antiferro-octupole order. By minimizing the elastic energy (4.18) associated with the $\Gamma_{5 g}$ moments, we obtain

$$
\epsilon_{\alpha \beta}=-\frac{g_{\Gamma_{5}}}{4 C_{44}^{(0)}} \sum_{i}\left\langle O_{\alpha \beta} i\right\rangle
$$

We use the following experimental values for $\mathrm{Ce}_{0.75} \mathrm{La}_{0.25} \mathrm{~B}_{6}:\left|g_{\Gamma_{5}}\right|=155 \mathrm{~K}, \quad C_{44}^{(0)} \simeq 8.2 \times$ $10^{11} \mathrm{erg} / \mathrm{cm}^{3},{ }^{11)}$ and the lattice constant $a=4.13 \AA$. At absolute zero, the magnitude of quadrupole moments, induced by the octupole order, is given by $\left|\left\langle O_{y z} i\right\rangle\right|=\left|\left\langle O_{z x} i\right\rangle\right|=\left|\left\langle O_{x y} i\right\rangle\right|=2 / 3$, and we obtain

$$
\left|\epsilon_{y z}\right|=\left|\epsilon_{z x}\right|=\left|\epsilon_{x y}\right|=4.6 \times 10^{-5} .
$$

In order to account for the observed lattice contraction along [111], ${ }^{13,14)}$ we consider two possibilities: (i) $g_{\Gamma_{5}}<0$ and (ii) $g_{\Gamma_{5}}>0$.

\section{1 case (i) $g_{\Gamma_{5}}<0$}

In case (i), we assume that a small stress which accompanies the measurement breaks the equivalence of four octupole domains, and chooses a domain for which the contraction becomes maximum along [111] with $g_{\Gamma_{5}}<0$. In this case, the domain with $\left\langle O_{y z} i\right\rangle=\left\langle O_{z x} i\right\rangle=$ $\left\langle O_{x y} i\right\rangle=-2 / 3$ is chosen, and all $\epsilon_{\alpha \beta}$ are negative. Then we obtain

$$
\Delta l / l=\frac{1}{3} \epsilon_{\mathrm{B}}+\frac{2}{3}\left(\epsilon_{y z}+\epsilon_{z x}+\epsilon_{x y}\right)=\frac{1}{3} \epsilon_{\mathrm{B}}+2 \epsilon_{x y},
$$

along [111], where $\epsilon_{\mathrm{B}}$ is the volume strain which is not included in our model. Experimentally, the value of the volume strain in $\mathrm{Ce}_{0.7} \mathrm{La}_{0.3} \mathrm{~B}_{6}$ was obtained to be $\epsilon_{\mathrm{B}}=$

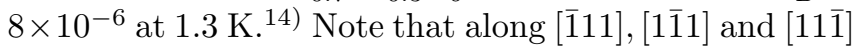
directions, the lattice should expand by

$$
\Delta l / l=\frac{1}{3} \epsilon_{\mathrm{B}}+\frac{2}{3}\left|\epsilon_{x y}\right| .
$$

\section{2 case (ii) $g_{\Gamma_{5}}>0$}

In case (ii), a positive stress along [111] may favor a domain which contracts along this direction. In this case, two of $\epsilon_{\alpha \beta}$ are negative and the other is positive. Then the contraction along [111] is given by,

$$
\Delta l / l=\frac{1}{3} \epsilon_{\mathrm{B}}+\frac{2}{3}\left(\epsilon_{y z}+\epsilon_{z x}+\epsilon_{x y}\right)=\frac{1}{3} \epsilon_{\mathrm{B}}-\frac{2}{3}\left|\epsilon_{x y}\right| .
$$

The crystal expands along another direction. In the single domain with $\left\langle O_{y z}\right\rangle=\left\langle O_{z x}\right\rangle=-\left\langle O_{x y}\right\rangle$, for example, an expansion along [11̄i] should be present, and it is given by

$$
\Delta l / l=\frac{1}{3} \epsilon_{\mathrm{B}}+\frac{2}{3}\left(-\epsilon_{y z}-\epsilon_{z x}+\epsilon_{x y}\right)=\frac{1}{3} \epsilon_{\mathrm{B}}+2\left|\epsilon_{x y}\right| .
$$

\subsection{Comparison with the Experiment}

According to an experiment, ${ }^{14)}$ the shear strain on the assumption of the trigonal symmetry around [111], i.e., case (i), is derived as $\epsilon_{\alpha \beta}=-4 \times 10^{-6}$ without a magnetic field at $1.3 \mathrm{~K}$. Assuming that the phase IV is stable down to zero temperature, $\epsilon_{\alpha \beta}$ is extrapolated to $-6 \times 10^{-6}$ to $-10 \times 10^{-6}$ at absolute zero. The absolute value is by an order of magnitude smaller than our estimate in eq. (5.40). Thus the observed reduction of $\left|\left\langle O_{\alpha \beta}\right\rangle\right|$ should come from quantum fluctuations neglected here.

Another possibility is that case (ii) is realized. In this case, the value of the strain becomes three times larger, i.e., $\left|\epsilon_{\alpha \beta}\right|=(2-3) \times 10^{-5}$, when deduced from the same experimental result. ${ }^{14)}$ This value is comparable to that given in eq. (5.40). As mentioned in $\S 3$, a state where one of $\left\langle O_{\alpha \beta}\right\rangle$ 's has a sign different from the others is stabilized under $\boldsymbol{H} \|$ [111] without an external stress. Experimentally the contraction is enhanced under $\boldsymbol{H} \|$ $[111] .{ }^{13,14)}$ This feature favors case (ii) in our model.

To determine which case is realized in the experiment, it is desirable to measure the lattice distortion along the [110] direction. The lattice distortion along [110] is given by $\Delta l / l=\left(\epsilon_{\mathrm{B}} / 3\right)+\epsilon_{x y}$. Thus, in case (i), our model predicts a smaller magnitude of lattice contraction along the [110] direction as compared with the contraction (5.41) along the [111] direction. On the other hand, in case (ii), the magnitude of lattice contraction along [110] is expected to be larger than that of the contraction (5.43) along [111].

\section{$5.4 N d B_{6}$}

For comparison, we estimate in the same manner the magnitude of the lattice distortion of $\mathrm{NdB}_{6}$. This material undergoes an A-type antiferromagnetic order at 
$T_{\mathrm{N}} \simeq 8 \mathrm{~K}^{28)}$ accompanying a lattice distortion along the [001] direction. ${ }^{29)}$ This lattice distortion indicates a $O_{2}^{0}$ ferro-quadrupole order. We estimate the lattice distortion in the $O_{2}^{0}$ ferro-quadrupole-ordered state by using the experimental values: $\left|g_{\Gamma_{3}}\right|=220 \mathrm{~K},\left(C_{11}-\right.$ $\left.C_{12}\right) / 2 \simeq 20 \times 10^{11} \mathrm{erg} / \mathrm{cm}^{3},{ }^{30)} a=4.12 \AA$, and the Lea-Leask-Wolf parameter $x_{\mathrm{LLW}}=-0.82 .{ }^{31)}$ We assume that $\left(C_{11}^{(0)}-C_{12}^{(0)}\right) / 2$ is almost the same as the observed $\left(C_{11}-C_{12}\right) / 2$. In a magnetic field $\boldsymbol{H} \|[100]$, a state with the antiferromagnetic moment perpendicular to [100] is stabilized. For a domain where the magnetic moment is along [001] or [010], we obtain

$$
|\Delta l| / l=2.8 \times 10^{-4}
$$

along $\boldsymbol{H}$, on the assumption $\epsilon_{\mathrm{B}}=0$. This value is favorably comparable to the experimental one $\Delta l / l \simeq$ $-2 \times 10^{-4}$ at $2 \mathrm{~K}$ and $H=2.1 \mathrm{~T}^{29)}$ The distortion in $\mathrm{NdB}_{6}$ is by an order of magnitude larger than those in eqs. (5.41) and (5.43). The reason is that the quadrupole moment $\left|\left\langle O_{2}^{0}\right\rangle\right|=4.5$ in $\mathrm{NdB}_{6}$ is much larger than the corresponding value $\left|\left\langle O_{x y}\right\rangle\right|=2 / 3$ in the octupolar state of $\mathrm{Ce}_{x} \mathrm{La}_{1-x} \mathrm{~B}_{6}$, while the magneto-elastic coupling constants are of the same order.

\section{Internal Field}

In this section, we discuss the internal magnetic field associated with the octupole order. The $\mu \mathrm{SR}$ time spectra in the phase IV consist of a Gaussian component and an exponential component. ${ }^{17)}$ The observation of a Gaussian relaxation indicates that internal fields are randomly distributed and/or fluctuating. The internal field deduced from the Gaussian relaxation is of the order of $0.1 \mathrm{~T}$. We discuss whether the octupole moment can be the origin of the Gaussian relaxation. In the $\mu \mathrm{SR}$ measurement, $\mu^{+}$locates at $(a / 2,0,0)$ and equivalent sites $^{32-34)}$ with a Ce ion chosen as the origin. As a reference the internal field from a Bohr magneton $\mu_{B}$ is estimated to be

$$
H_{\text {dipole }}=\frac{1}{(a / 2)^{3}} \mu_{\mathrm{B}} \simeq 0.1 \mathrm{~T},
$$

with $a / 2 \simeq 2 \AA$. The internal field from an octupole with the size $r$ is estimated to be

$$
H_{\text {octupole }}=\frac{r^{2}}{(a / 2)^{5}} \mu_{\mathrm{B}} \simeq 0.01 \mathrm{~T},
$$

with $r \simeq a_{B}=0.53 \AA$. For a more accurate estimate, we consider the multipole expansion of the vector potential from local electrons, which is given by ${ }^{35)}$

$$
\boldsymbol{A}(\boldsymbol{r})=\sum_{k, m} \frac{-\mathrm{i}}{k} r^{-(k+1)}\left(\boldsymbol{l} C_{m}^{(k)}(\theta, \phi)\right) M_{k}^{m},
$$

where $\boldsymbol{l}$ is the orbital angular momentum operator, $C_{m}^{(k)}(\theta, \phi)$ is $\sqrt{4 \pi /(2 k+1)}$ times the spherical harmonics $Y_{k m}(\theta, \phi)$, and $M_{k}^{m}$ is the magnetic multipole moment. The multipole moment is determined by the wave function $\psi_{i}(\boldsymbol{r})$ of the $i$-th electron, and the orbital and spin angular momentum operators $\boldsymbol{l}_{i}$ and $\boldsymbol{s}_{i}$. Namely we have

$$
\begin{aligned}
M_{k}^{m}= & \mu_{\mathrm{B}} \sum_{i} \int \mathrm{d} \boldsymbol{r}_{i} \psi_{i}^{*}\left(\boldsymbol{r}_{i}\right)\left(\boldsymbol{\nabla}_{i} r_{i}^{k} C_{m}^{(k) *}\left(\theta_{i}, \phi_{i}\right)\right) \\
& \cdot\left(\frac{2}{k+1} \boldsymbol{l}_{i}+2 \boldsymbol{s}_{i}\right) \psi_{i}\left(\boldsymbol{r}_{i}\right) .
\end{aligned}
$$

Equation (6.49) is evaluated with the use of the operator equivalents method. ${ }^{36)}$ For our purpose, it is sufficient to consider only octupole moments. Then we obtain $M_{3}^{m}$ through calculation of the reduced matrix element of the third-rank tensor. The result for one-electron states with $J=5 / 2, L=3, S=1 / 2$ is given by

$$
M_{3}^{m}=-\frac{2}{35} \mu_{\mathrm{B}}\left\langle r^{2}\right\rangle\left\langle J_{m}^{(3)}\right\rangle,
$$

where the third-rank tensor operators $J_{m}^{(3)}$ are defined by

$$
\begin{aligned}
J_{3}^{(3)} & =-\frac{\sqrt{5}}{4}\left(J_{x}+\mathrm{i} J_{y}\right)^{3}, \\
J_{m-1}^{(3)} & =\frac{1}{\sqrt{(3+m)(4-m)}}\left[J_{x}-\mathrm{i} J_{y}, J_{m}^{(3)}\right] .
\end{aligned}
$$

We introduce linear combinations:

$$
\begin{aligned}
M_{3}^{2 u} & =\frac{1}{\sqrt{2}}\left[M_{3}^{+2}-M_{3}^{-2}\right], \\
M_{3}^{4 u x} & =\frac{1}{4}\left[-\sqrt{5}\left(M_{3}^{+3}-M_{3}^{-3}\right)+\sqrt{3}\left(M_{3}^{+1}-M_{3}^{-1}\right)\right], \\
M_{3}^{4 u y} & =\frac{1}{4 \mathrm{i}}\left[\sqrt{5}\left(M_{3}^{+3}+M_{3}^{-3}\right)+\sqrt{3}\left(M_{3}^{+1}+M_{3}^{-1}\right)\right], \\
M_{3}^{4 u z} & =M_{3}^{0}, \\
M_{3}^{5 u x} & =\frac{1}{4}\left[\sqrt{3}\left(M_{3}^{+3}-M_{3}^{-3}\right)+\sqrt{5}\left(M_{3}^{+1}-M_{3}^{-1}\right)\right], \\
M_{3}^{5 u y} & =\frac{1}{4 \mathrm{i}}\left[\sqrt{3}\left(M_{3}^{+3}+M_{3}^{-3}\right)-\sqrt{5}\left(M_{3}^{+1}+M_{3}^{-1}\right)\right], \\
M_{3}^{5 u z} & =\frac{1}{\sqrt{2}}\left[M_{3}^{+2}+M_{3}^{-2}\right] .
\end{aligned}
$$

We then obtain $\boldsymbol{H}=\boldsymbol{\nabla} \times \boldsymbol{A}$ as

$$
\begin{aligned}
H_{z}(\boldsymbol{r})=\frac{1}{36 r^{5}} & {\left[6 \sqrt{15} O_{x y}(\boldsymbol{r})\left(7 \sqrt{3} O_{2}^{0}(\boldsymbol{r})+4\right) M_{3}^{2 u}\right.} \\
& +15 O_{z x}(\boldsymbol{r})\left(7 \sqrt{3} O_{2}^{0 x}(\boldsymbol{r})-2\right) M_{3}^{4 u x} \\
& +15 O_{y z}(\boldsymbol{r})\left(7 \sqrt{3} O_{2}^{0 y}(\boldsymbol{r})-2\right) M_{3}^{4 u y} \\
& +2\left(105 O_{2}^{0}(\boldsymbol{r}) O_{2}^{0}(\boldsymbol{r})-20 \sqrt{3} O_{2}^{0}(\boldsymbol{r})-28\right) M_{3}^{4 u z} \\
& +9 \sqrt{15} O_{z x}(\boldsymbol{r})\left(7 O_{2}^{2 x}(\boldsymbol{r})+2\right) M_{3}^{5 u x} \\
& +9 \sqrt{15} O_{y z}(\boldsymbol{r})\left(7 O_{2}^{2 y}(\boldsymbol{r})-2\right) M_{3}^{5 u y} \\
& \left.+6 \sqrt{15} O_{2}^{2}(\boldsymbol{r})\left(7 \sqrt{3} O_{2}^{0}(\boldsymbol{r})+4\right) M_{3}^{5 u z}\right]
\end{aligned}
$$


where

$$
\begin{aligned}
& O_{2}^{0 x}(\boldsymbol{r})=\left(2 x^{2}-y^{2}-z^{2}\right) /\left(\sqrt{3} r^{2}\right), \\
& O_{2}^{0 y}(\boldsymbol{r})=\left(2 y^{2}-z^{2}-x^{2}\right) /\left(\sqrt{3} r^{2}\right), \\
& O_{2}^{2 x}(\boldsymbol{r})=\left(y^{2}-z^{2}\right) / r^{2} \\
& O_{2}^{2 y}(\boldsymbol{r})=\left(z^{2}-x^{2}\right) / r^{2}
\end{aligned}
$$

and the other $O(\boldsymbol{r})$ 's are obtained by replacing $J_{\alpha}$ in eqs. (2.8)-(2.10) with $\alpha / r$. The other components $H_{x}(\boldsymbol{r})$ and $H_{y}(\boldsymbol{r})$ are obtained by exchanging $x, y, z$ cyclically from above. In the $\Gamma_{5}$-ordered state, we obtain $M_{3}^{5 u x}=$ $M_{3}^{5 u y}=M_{3}^{5 u z} \neq 0$, and eqs. (6.53)-(6.56) are zero.

To estimate the internal magnetic field, we use the value $\left\langle r^{2}\right\rangle=1.298$ in atomic unit, which was obtained with a relativistic Dirac-Fock calculation by Freeman and Desclaux. ${ }^{37)}$ Then we obtain about $40 \mathrm{G}$ as the internal field at $(a / 2,0,0)$ from a Ce ion. This value is by an order of magnitude smaller than that derived by the $\mu \mathrm{SR}$ measurement. ${ }^{17)}$ Thus the static octupole moment alone cannot account for the Gaussian relaxation of the $\mu \mathrm{SR}$ spectra. In a future work, we plan to study in more detail fluctuations in the octupole-ordered state. If the origin of the $\mu \mathrm{SR}$ spectra is not an effect of fluctuations, we may have to consider a disturbance of the ordered state by muons.

We mention that Paixão et al. have proposed for the ordered state of $\mathrm{NpO}_{2}$ that a triple- $\boldsymbol{q} \Gamma_{5 u}$-type octupole ordering is realized and a triple- $\boldsymbol{q} \Gamma_{5 g}$ quadrupole moment is induced. ${ }^{2)}$ From the observed muon spin precession frequency of $\omega=7 \mathrm{MHz}^{38}$ ) and the muon gyromagnetic ratio $\gamma_{\mu}=2 \pi \times 13.55 \mathrm{MHz} / \mathrm{kG}$ at $8 \mathrm{~K}$, we estimate the internal magnetic field as $H=\omega / \gamma_{\mu} \simeq 500$ G. $^{39}$ ) This internal field is smaller than that of antiferromagnetic $\mathrm{UO}_{2}$ by an order of magnitude. Thus the observed field in $\mathrm{NpO}_{2}$ may be interpreted as coming from octupole moments. However, the internal field in $\mathrm{NpO}_{2}$ is still much larger than that of eq. (6.47). Provided the muon is really probing the intrinsic internal field of the systems, the muon stopping site should be about $1 \AA$ from a $\mathrm{Np}$ ion.

\section{Pressure Induced Moment}

It has been discussed that antiferromagnetism should be induced in an antiferro-octupolar phase by uniaxial stress. ${ }^{5}$ We apply this idea to the case of the $\Gamma_{5 u}$ order. The uniaxial pressure $p$ along the [001] direction accompanies the $\Gamma_{3}$ strain

$$
\epsilon_{u}=\frac{1}{\sqrt{3}}\left(2 \epsilon_{z z}-\epsilon_{x x}-\epsilon_{y y}\right)=\frac{p}{\sqrt{3}\left(C_{11}-C_{12}\right) / 2} .
$$

We solve the mean field equation with the finite strain $\epsilon_{u}$ and the corresponding quadrupole-strain interaction $g_{\Gamma_{3}}$ (see, eq. (4.18)). The antiferromagnetic moment is induced in the $x y$-plane since $\left\langle T_{x}^{5 u}\right\rangle \neq 0$ together with $\left\langle J_{y}^{2}-J_{z}^{2}\right\rangle \neq 0$ gives $\left\langle J_{x}\right\rangle \neq 0$. The direction of the antiferromagnetic moment is along [110] or [110]. In Figs. 9 and 10 , we show the pressure dependence of the octupole and magnetic moments at absolute zero with experimental values: $\left(C_{11}-C_{12}\right) / 2 \simeq 20.4 \times 10^{11} \mathrm{erg} / \mathrm{cm}^{3}$ and $\left|g_{\Gamma_{3}}\right|=120 \mathrm{~K} .{ }^{11)} \quad$ The mean field solution for $\left\langle T_{z}^{5 u}\right\rangle$ be-

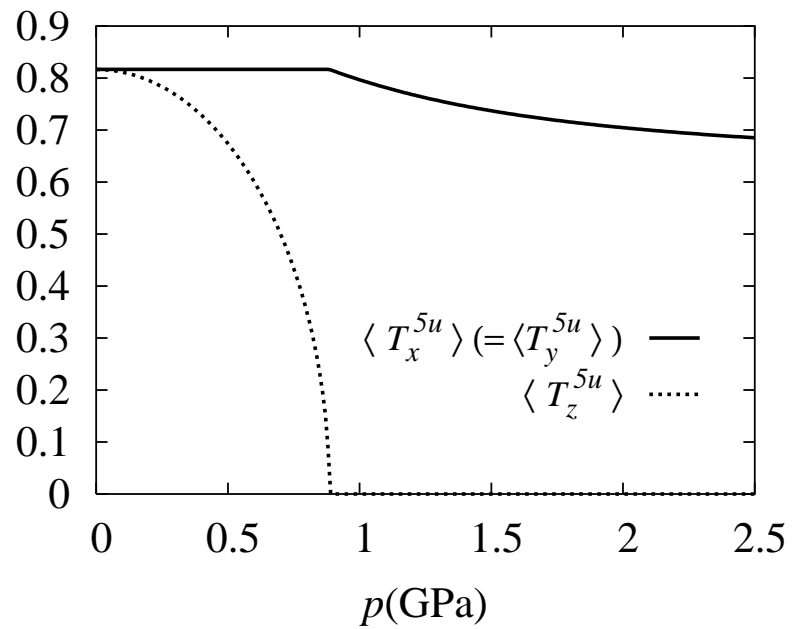

Fig. 9. Pressure dependence of the antiferro-octupole moment $\left\langle T_{x}^{5 u}\right\rangle\left(=\left\langle T_{y}^{5 u}\right\rangle\right)$ and $\left\langle T_{z}^{5 u}\right\rangle$.

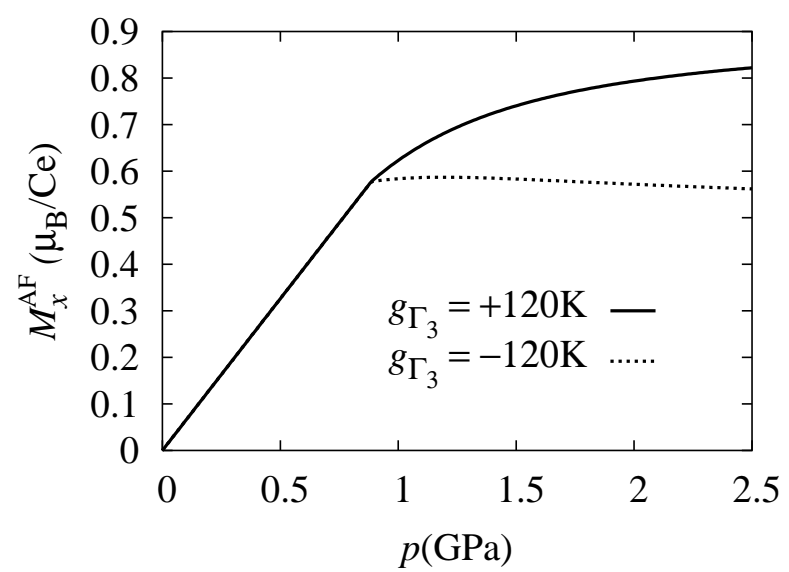

Fig. 10. Pressure dependence of the antiferromagnetic moment $M_{x}^{\mathrm{AF}}\left(=M_{y}^{\mathrm{AF}}\right) \cdot M_{z}^{\mathrm{AF}}=0$.

comes zero for $p \geq 0.9 \mathrm{GPa}$. This implies that both the octupole and dipole moments lie in the $x y$-plane. With $p=1 \mathrm{GPa}$, the magnetic moment is estimated to be $0.88 \mu_{\mathrm{B}}\left(0.82 \mu_{\mathrm{B}}\right)$, when $g_{\Gamma_{3}}$ is positive (negative). This value should actually be reduced by quantum fluctuations neglected here. However, it is likely that this value remains within the experimental range.

\section{Summary}

To summarize, we have proposed that the $\Gamma_{5 u}$ octupole moment is a plausible candidate for the order parameter of the phase IV in $\mathrm{Ce}_{x} \mathrm{La}_{1-x} \mathrm{~B}_{6}$. This octupole ordered state is consistent with the lattice distortion along the [111] direction, ${ }^{13,14)}$ the softening of the elastic constant $C_{44},{ }^{11)}$ the broken time reversal symmetry, ${ }^{16,17)}$ the absence of dipole moment, ${ }^{18)}$ and the cusp in the magnetization at $T_{\mathrm{I}-\mathrm{IV}} \cdot{ }^{12)}$ Especially, the sudden softening of $C_{44}$ and the cusp in the magnetization at $T_{\text {I-IV }}$ are difficult to be explained by a pure quadrupole order, but are reproduced by the present octupole ordering model.

Many properties of the phase IV are consistent with the octupole order, but the internal field estimated by our 
mean field theory is much smaller than that suggested by the $\mu \mathrm{SR}$ experiment. ${ }^{17)}$ We recall that the NMR spectra become broad in the phase IV, ${ }^{16}$ ) and the spectra cannot be explained by the static and staggered octupole moments, either. Hence some dynamical or quantum fluctuation effects may be playing a significant role. Clarifying dynamical aspects and identifying the periodicity in the phase IV are the most challenging open problems to be addressed in the near future.

We have also shown that antiferromagnetic moment lying in the $x y$-plane is induced under uniaxial pressure along the $z$ direction. The estimated value of the antiferromagnetic moment is of the order of $1 \mu_{\mathrm{B}}$, and we wait for experimental efforts to detect the moment.

\section{Acknowledgements}

We thank Prof. A. Schenck for pointing out our mistake in the estimation of the internal field in $\mathrm{NpO}_{2}$. This work was supported partly by Special Coordination Funds for Promoting Science and Technology, and by the NEDO international collaboration program "New boride materials".

1) P. Santini and G. Amoretti: Phys. Rev. Lett. 85 (2000) 2188.

2) J. A. Paixão, C. Detlefs, M. J. Longfield, R. Caciuffo, P. Santini, N. Bernhoeft, J. Rebizant and G. H. Lander: Phys. Rev. Lett. 89 (2002) 187202.

3) R. Caciuffo, J. A. Paixão, C. Detlefs, M. J. Longfield, P. Santini, N. Bernhoeft, J. Rebizant and G. H. Lander: J. Phys.: Condens. Matter 15 (2003) S2287.

4) A. Kiss and P. Fazekas: cond-mat/0306215.

5) Y. Kuramoto and H. Kusunose: J. Phys. Soc. Jpn. 69 (2000) 671.

6) H. Kusunose and Y. Kuramoto: J. Phys. Soc. Jpn. 70 (2001) 1751.

7) R. Siina, H. Shiba and P. Thalmeier: J. Phys. Soc. Jpn. 66 (1997) 1741.

8) O. Sakai, R. Siina, H. Shiba and P. Thalmeier: J. Phys. Soc. Jpn. 66 (1997) 3005.

9) R. Siina, O. Sakai, H. Shiba and P. Thalmeier: J. Phys. Soc. Jpn. 67 (1998) 941.

10) T. Sakakibara, T. Tayama, H. Amitsuka, K. Tenya, S. Kunii, T. Suzuki and A. Ochiai: Physica B 230-232 (1997) 307.

11) O. Suzuki, T. Goto, S. Nakamura, T. Matsumura and S. Kunii: J. Phys. Soc. Jpn. 67 (1998) 4243.

12) T. Tayama, T. Sakakibara, K. Tenya, H. Amitsuka and S. Kunii: J. Phys. Soc. Jpn. 66 (1997) 2268.

13) M. Akatsu, O. Suzuki, Y. Nemoto, T. Goto, S. Nakamura and S. Kunii: Proc. Int. Conf. Strongly Correlated Electrons with Orbital Degrees of Freedom (ORBITAL2001), J. Phys. Soc. Jpn. 71 (2002) Suppl., p. 115.

14) M. Akatsu, T. Goto, Y. Nemoto, O. Suzuki, S. Nakamura and
S. Kunii: J. Phys. Soc. Jpn. 72 (2003) 205.

15) T. Furuno, N. Sato, S. Kunii, T. Kasuya and W. Sasaki: J. Phys. Soc. Jpn. 54 (1985) 1899.

16) K. Magishi, M. Kawakami, T. Saito, K. Koyama, K. Mizuno and S. Kunii: Z. Naturforsch. 57 a (2002) 441.

17) H. Takagiwa, K. Ohishi, J. Akimitsu, W. Higemoto, R. Kadono, M. Sera and S. Kunii: J. Phys. Soc. Jpn. 71 (2002) 31.

18) K. Iwasa, K. Kuwahara, M. Kohgi, P. Fischer, A. Dönni, L. Keller, T. C. Hansen, S. Kunii, N. Metoki, Y. Koike and K. Ohoyama: Physica B 329-333 (2003) 582.

19) T. Sakakibara, K. Tenya, M. Yokoyama, H. Amitsuka and S. Kunii: Proc. Int. Conf. Strongly Correlated Electrons with Orbital Degrees of Freedom (ORBITAL2001), J. Phys. Soc. Jpn. 71 (2002) Suppl., p. 48.

20) T. Sakakibara, T. Tayama, K. Tenya, M. Yokoyama, H. Amitsuka, D. Aoki, Y. Ōnuki, Z. Kletowski and S. Kunii: J. Phys. Chem. Solids 63 (2002) 1147.

21) T. Morie, T. Onimaru, T. Tayama, T. Sakakibara and S. Kunii: presented at the autumn meeting of the Phys. Soc. Jpn. (2003).

22) K. Kubo and Y. Kuramoto: J. Phys. Soc. Jpn. 72 (2003) 1859.

23) E. Zirngiebl, B. Hillebrands, S. Blumenröder, G. Güntherodt, M. Loewenhaupt, J. M. Carpenter, K. Winzer and Z. Fisk: Phys. Rev. B 30 (1984) 4052.

24) B. Lüthi, S. Blumenröder, B. Hillebrands, E. Zirngiebl, G. Güntherodt and K. Winzer: Z. Phys. B 58 (1984) 31.

25) N. Sato, S. Kunii, I. Oguro, T. Komatsubara and T. Kasuya: J. Phys. Soc. Jpn. 53 (1984) 3967.

26) P. Morin, A. Waintal and B. Lüthi: Phys. Rev. B 14 (1976) 2972.

27) See, for example, B. Callen: THERMODYNAMICS (John Wiley \& Sons, 1960)

28) C. M. McCarthy and C. W. Tompson: J. Phys. Chem. Solids 41 (1980) 1319.

29) M. Sera, S. Itabashi and S. Kunii: J. Phys. Soc. Jpn. 66 (1997) 548.

30) A. Tamaki, T. Goto, M. Yoshizawa, T. Fujimura, S. Kunii and T. Kasuya: J. Magn. Magn. Mater. 52 (1985) 257.

31) G. Pofahl, E. Zirngiebl, S. Blumenröder, H. Brenten, G. Güntherodt and K. Winzer: Z. Phys. B 66 (1987) 339.

32) A. Amato, R. Feyerherm, F. N. Gygax and A. Schenck: Hyperfine Interact. 104 (1997) 115.

33) R. Kadono, W. Higemoto, A. Koda, K. Kakuta, K. Ohishi, H. Takagiwa, T. Yokoo and J. Akimitsu: J. Phys. Soc. Jpn. 69 (2000) 3189.

34) A. Schenck, F. N. Gygax and S. Kunii: Phys. Rev. Lett. 89 (2002) 037201.

35) C. Schwartz: Phys. Rev. 97 (1955) 380.

36) See, for example, T. Inui, Y. Tanabe and Y. Onodera: Group Theory and Its Applications in Physics (Springer-Verlag, Berlin, 1996) 2nd ed.

37) A. J. Freeman and J. P. Desclaux: J. Magn. Magn. Mater. 12 (1979) 11.

38) W. Kopmann, F. J. Litterst, H.-H. Klauß, M. Hillberg, W. Wagener, G. M. Kalvius, E. Schreier, F. J. Burghart, J. Rebizant and G. H. Lander: J. Alloys Compounds 271-273 (1998) 463.

39) In our previous paper, ${ }^{22)}$ we have mistaken the estimation of the internal field in $\mathrm{NpO}_{2}$ by the factor of $2 \pi$. 